\title{
Binary Symmetric Matrix Inversion through Local Complementation
}

\author{
Robert Brijder \\ Hasselt University and Transnational University of Limburg \\ Belgium
}

Hendrik Jan Hoogeboom

Leiden Institute of Advanced Computer Science

Leiden University, The Netherlands

\begin{abstract}
We consider the Schur complement operation for symmetric matrices over GF(2), which we identify with graphs through the adjacency matrix representation. It is known that Schur complementation for such a matrix (i.e., for a graph) can be decomposed into a sequence of two types of elementary Schur complement operations: (1) local complementation on a looped vertex followed by deletion of that vertex and (2) edge complementation on an edge without looped vertices followed by deletion of that edge. We characterize the symmetric matrices over GF(2) that can be transformed into the empty matrix using only operations of (1). As a consequence, we find that these matrices can be inverted using local complementation. The result is applied to the theory of gene assembly in ciliates.
\end{abstract}

\section{Introduction}

Principal pivot transform (called pivot for short) is an operation on matrices that partially, for a subset of the components, inverts a given matrix [12]. The operation of Schur complementation [13] is pivot followed by the deletion of the rows and columns involved in this pivot. Pivot and Schur complementation are defined on matrices over arbitrary fields, but here we consider symmetric matrices over the two-element field $\mathbb{F}_{2}$, which we identify with undirected graphs (where loops are allowed) through the adjacency matrix representation. In this case, a pivot operation can be decomposed into a sequence of two types of pivot operations, called local complementation and edge complementation [9]. Local complementation is applied to a looped vertex, and edge complementation is applied to an unlooped edge. Both graph operations toggle particular edges in the neighbourhood of the vertex/edge involved. The Schur complementations corresponding to the pivot operations of local complementation and edge complementation are called vertex reduction and edge reduction, respectively. 
The process of unscrambling of genes in ciliates can be modelled using graphs and three (types of) graph rewriting rules, recalled here briefly in Chapter 4, but covered extensively in the book [6]. It is observed by Harju et al. [3] that vertex and edge reduction happen to correspond to two of these graph operations. The third operation of this model simply deletes remaining isolated unlooped vertices. In this model, the graphs have been characterized that can be transformed to the empty graph using this third operation and any subset of the remaining two graph operations [7, 6]. It remained an open question to obtain a characterization when allowing only the first two operations (each alone, or in combination).

In this paper, we characterize the graphs that can be transformed to the empty graph using vertex or edge reduction. Since a transformation using Schur complementation to the empty graph corresponds to a (full) matrix inversion using pivot, we characterize in this way the graphs that can be transformed to the empty graph by applying local or edge complementation on each vertex exactly once. Also, in this way we extend the result of $[7,6]$ to obtain a characterization of the graphs that can be transformed to the empty graph using any subset of the three graph operations. Although we need an algebraic background to understand the interplay between the separate operations, it is quite surprising that the proof of the main result instead uses combinatorial techniques.

\section{Pivots}

The principal pivot transform, or pivot for short, is defined for matrices over arbitrary fields, see, e.g., [11] for an overview. We start by giving the general definition, and then we consider symmetric matrices over the two-element field $\mathbb{F}_{2}$. In this case, pivot can be seen as a particular graph operation.

We consider $V \times V$-matrices, i.e., matrices where the rows and columns are indexed by a finite set $V$ (formally, a $V \times V$-matrix is a function $V^{2} \rightarrow F$ where $F$ is some field). Since we do not fix an ordering of $V$, the rows and columns of the matrices are not ordered. Observe that determinant of such a matrix is well defined, since one may choose the linear ordering of $V$, i.e., the bijection $V \rightarrow\{1, \ldots,|V|\}$, arbitrarily.

Matrices. Let $A$ be a $V \times V$-matrix (over an arbitrary field), and let $X \subseteq V$ be such that the principal submatrix $A[X]$ induced by $X$ is nonsingular, i.e., $\operatorname{det} A[X] \neq 0$. The pivot of $A$ on $X$, denoted by $A * X$, is defined as follows [12]. If $P=A[X]$ and we decompose $A$ accordingly as $A=\left(\begin{array}{cc}P & Q \\ R & S\end{array}\right)$, then

$$
A * X=\left(\begin{array}{cc}
P^{-1} & -P^{-1} Q \\
R P^{-1} & S-R P^{-1} Q
\end{array}\right)
$$

The pivot can be considered a partial inverse, as $A$ and $A * X$ satisfy the following relation, where the vectors $x_{1}$ and $x_{2}$ correspond to the elements of $X$ (see [11]).

$$
A\left(\begin{array}{l}
x_{1} \\
y_{1}
\end{array}\right)=\left(\begin{array}{l}
x_{2} \\
y_{2}
\end{array}\right) \text { iff } A * X\left(\begin{array}{l}
x_{2} \\
y_{1}
\end{array}\right)=\left(\begin{array}{l}
x_{1} \\
y_{2}
\end{array}\right)
$$

This relation can in fact be used to define $A * X$ given $A$ and $X$, and many important properties can be obtained elegantly using this characteristic relation. Observe that $A * V=A^{-1}$ when $\operatorname{det} A \neq 0$. Also note that a pivot operation is an involution (operation of order 2), and more generally, if $(A * X) * Y$ is defined, then $A *(X \oplus Y)$ is defined and they are equal. 

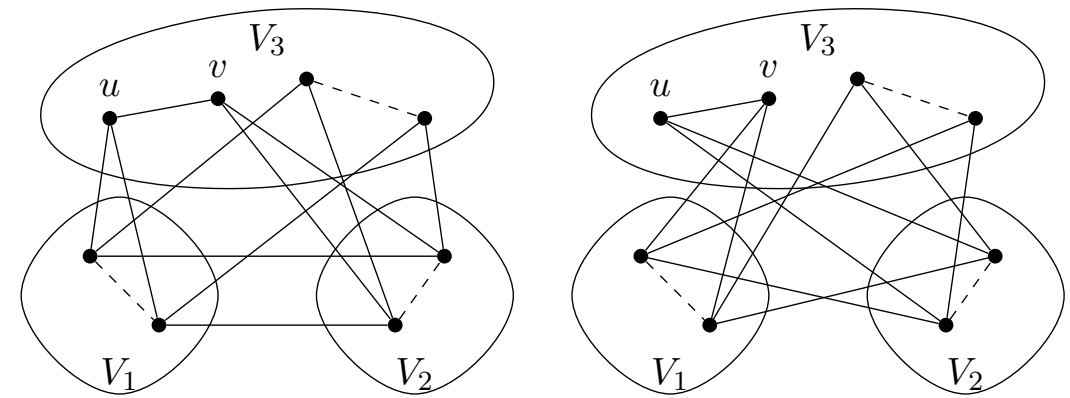

Figure 1. Pivot on an edge $\{u, v\}$ in a graph. Adjacency between vertices $x$ and $y$ is toggled iff $x \in V_{i}$ and $y \in V_{j}$ with $i \neq j$. Note that $u$ and $v$ are adjacent to all vertices in $V_{3}$ - these edges are omitted in the diagram. The operation does not affect edges adjacent to vertices outside the sets $V_{1}, V_{2}, V_{3}$, nor does it change any of the loops.

Schur complement. Removing the columns and rows of the matrix corresponding to the elements to which pivot was applied, corresponds to retaining the lower-right block $(A * X)[V \backslash X]=S-R P^{-1} Q$ in the definition of pivot, see equation (1). This block is known as the Schur complement (of $X$ in $A$ ), which we denote by $A / X$. It arises in the context of many matrix applications, e.g., solving linear equations, probability theory and statistics. It enjoys many surprising properties, see [13] for an extensive background.

We highlight now two of these properties for further use. The rank additivity formula $\operatorname{rank}(A)=$ $\operatorname{rank}(A[X])+\operatorname{rank}(A / X)$ implies that the nullity of the Schur complement $A / X$ equals that of the original matrix $A$ (since $\operatorname{rank}(A[X])=|X|)$. In particular, this implies that $A / X$ is nonsingular when $A$ is. The sequential property of Schur complementation states that consecutive operations may be composed. More specifically, $(A / X) / Y=A /(X \cup Y)$, for disjoint $X, Y \subseteq V$ such that the operations are defined, i.e., $A[X]$ and $A[X \cup Y]$ are nonsingular.

Graphs. In this paper we restrict principal pivot transform and Schur complementation to symmetric matrices $A$ over $\mathbb{F}_{2}$. In this setting, $A$ may be identified with a graph $G$, where $A$ is the adjacency matrix representation of $G$, denoted by $A(G)$. Thus, graphs in this paper are undirected with loops allowed. As we identify $G$ with $A(G)$, notions and notation regarding matrices are carried over to graphs and vice versa. For example, by the rank of $G$ we mean the rank of $A(G)$, and for $X \subseteq V$, the subgraph $G[X]$ induced by $X$ is the graph with adjacency matrix $A(G)[X]$.

Geelen [9] observes (by extending an observation of Bouchet [1] concerning simple graphs) that any pivot on a graph $G$ can be decomposed into a sequence of elementary pivots that are either of the form $G *\{u\}$ where $u$ is a looped vertex, or of the form $G *\{u, v\}$ where $\{u, v\}$ is an edge and vertices $u$ and $v$ are both non-looped vertices. Thus, for a general pivot operation on graphs it suffices to consider these two cases.

The elementary pivot $G *\{u\}$ on a loop $\{u\}$ is called local complementation. It is the graph obtained from $G$ by "toggling" the edges in the neighbourhood $N_{G}(u)=\{v \in V \mid\{u, v\} \in E(G), u \neq v\}$ of $u$ in $G$ : for each $v, w \in N_{G}(u),\{v, w\} \in E(G)$ iff $\{v, w\} \notin E(G *\{u\})$ (allowing $v=w$ ). The other edges are left unchanged. 


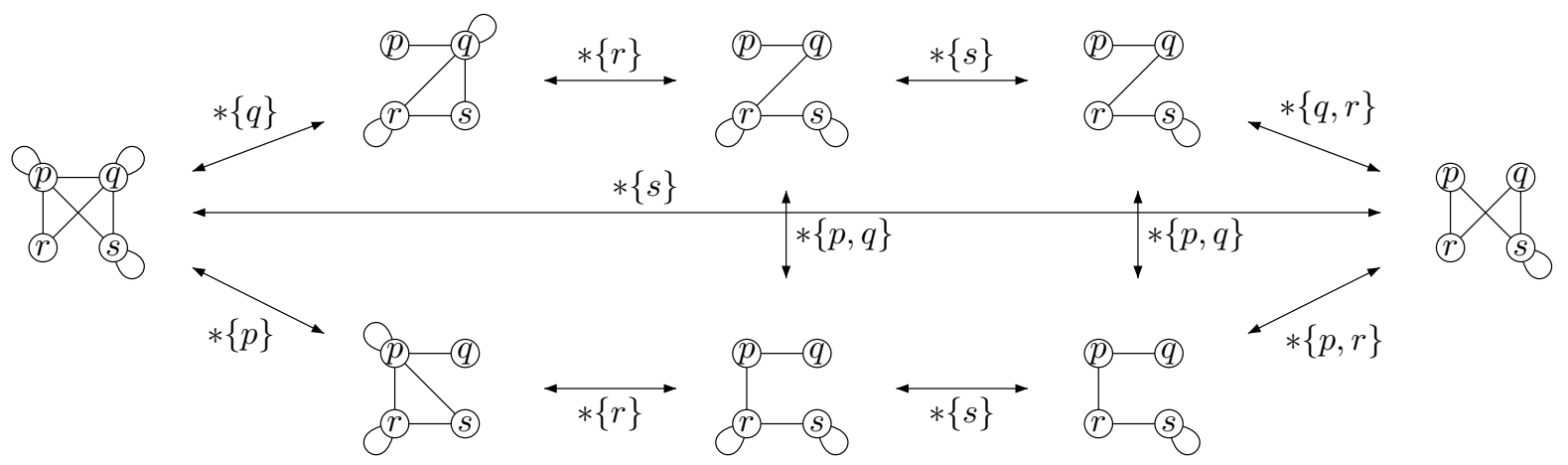

Figure 2. An orbit of graphs under pivot. Only the elementary pivots are shown.

We now recall edge complementation $G *\{u, v\}$ on an edge $\{u, v\}$ between non-loop vertices. For a vertex $x$ consider its closed neighbourhood $N_{G}^{\prime}(x)=N_{G}(x) \cup\{x\}$. The edge $\{u, v\}$ partitions the vertices of $G$ adjacent to $u$ or $v$ into three sets $V_{1}=N_{G}^{\prime}(u) \backslash N_{G}^{\prime}(v), V_{2}=N_{G}^{\prime}(v) \backslash N_{G}^{\prime}(u)$, $V_{3}=N_{G}^{\prime}(u) \cap N_{G}^{\prime}(v)$. Note that $u, v \in V_{3}$. The graph $G *\{u, v\}$ is constructed by "toggling" all edges between different $V_{i}$ and $V_{j}$ : for $\{x, y\}$ with $x \in V_{i}$ and $y \in V_{j}(i \neq j):\{x, y\} \in E(G)$ iff $\{x, y\} \notin E(G *\{u, v\})$, see Figure 1. The other edges remain unchanged. Note that, as a result of this operation, the neighbourhoods of $u$ and $v$ are interchanged.

Example 2.1. Consider the graph $G$ over $V=\{p, q, r, s\}$ with adjacency matrix

$$
A=\begin{aligned}
& p \\
& p \\
& q \\
& q \\
& s
\end{aligned}\left(\begin{array}{llll}
0 & 1 & 0 & 0 \\
1 & 1 & 1 & 1 \\
0 & 1 & 1 & 1 \\
0 & 1 & 1 & 0
\end{array}\right) .
$$

Then $G$ is depicted in Figure 2, top-row second column, together with its orbit under pivot. Only elementary pivots are given. All other pivots can be found by following a path in the diagram and taking the symmetric difference over the labels. As an example, the graph below $G$ equals $G *\{q\} *\{p\}=G *\{p, q\}$, but the pivot $*\{p, q\}$ of $G$ is not elementary: it is not on an unlooped edge.

The initial graph $G$ can be inverted using successive local complement operations, walking counterclockwise, leading to the inverse $G^{-1}$ given on the bottom-row fourth column.

The orbit of $G$ does not change (in this case) if we restrict to local complementation only; removal of the edge pivots results in a connected diagram. Hence, each of the edge complement operations can be replaced (in this case) by a sequence of local complementations, where operations may be repeated. For example, the middle two graphs in the top and bottom row of the figure, $G *\{r\}$ and $G *\{p, q, r\}$, are related by the edge pivot $*\{p, q\}$, but also by the sequence $*\{r\} *\{q\} *\{p\} *\{r\}$.

It is instructive to observe that nonsingularity of a graph is not maintained when applying pivot (thus neither are rank nor nullity). The graph $G$ under consideration can be inverted, while $G *\{q\}$ is singular; this can be seen by observing that the columns for $p$ and $q$ in the adjacency matrix for $G *\{q\}$ are equal. 


\section{Schur complement through local complementation}

We will now consider pivot $G * X$ for graphs, followed by deletion of the vertices in $X$, i.e., the Schur complement $G / X$. In particular we study Schur complementation $G /\{u\}$ on looped vertices and Schur complementation $G /\{u, v\}$ on edges with unlooped vertices. These operations are called vertex reduction and edge reduction, respectively.

In general, a sequence of Schur complementations is called a reduction. A reduction $\varphi$ is successful for a graph $G$ if $G \varphi$ is the empty graph (i.e., the graph with empty vertex set). We say that a graph $G$ is successfully reducible (using vertex/edge reduction) if there is a successful reduction $\varphi$ of $G$ (where $\varphi$ consists of only vertex/edge reductions, resp.). It follows from the discussion on elementary graph pivot that a graph $G$ is successfully reducible iff $G$ is nonsingular. If we allow either only vertex reduction or only edge reduction, then we can reduce only a proper subset of all nonsingular graphs.

Theorem 3.1. Let $A$ be a symmetric matrix over $\mathbb{F}_{2}$. Then $A$ is successfully reducible using edge reduction iff $A$ is nonsingular and has no 1 on the diagonal. Equivalently, a graph $G$ is successfully reducible using edge reduction iff $G$ is nonsingular and has no loops.

\section{Proof:}

As we have seen, the condition of nonsingularity is required for $A$ to be successfully reducible. According to Geelen [9] the pivot $A * V$ can be decomposed into a sequence of local complement and edge complement operations. The characterization now follows from the observation that edge complementation can only be applied to unlooped vertices and never introduces loops, while local complementation requires a looped vertex.

Obviously, if a graph is successfully reducible using edge reduction, then its number of vertices must be even. Curiously, this is implicit in the nonsingularity condition of Theorem 3.1. Indeed, nonsingular loop-free graphs must have at least one (actually an odd number of) so-called perfect matching (i.e., a complete pairing of vertices via a subset of edges), so the requirement is implied. For an explanation of the relationship between the determinant over $\mathbb{F}_{2}$ and perfect matchings see, e.g., [3].

A nonempty principal submatrix $A[X]$ of a matrix $A$ is called a component of $A$ if $A=\left(\begin{array}{cc}A[X] & 0 \\ 0 & A[V \backslash X]\end{array}\right)$, where the blocks indicated by 0 are null matrices of suitable size. Component $A[X]$ is called a connected component if $X$ is minimal with this property (w.r.t. inclusion), i.e., if $A[Y]$ is a component with $Y \subseteq X$, then $Y=X$. Again, we may carry this notation over to graphs. Consequently, $G[X]$ is a component of $G$ iff there are no edges between $X$ and $V \backslash X$ in $G$, and the notion of a connected component of $G$ is the usual graph-theoretic notion of a connected component of a graph $G$.

Theorem 3.2. Let $A$ be a symmetric matrix over $\mathbb{F}_{2}$. Then $A$ is successfully reducible using vertex reduction iff $A$ is nonsingular and each component of $A$ has at least one 1 on the diagonal. Equivalently, a graph $G$ is successfully reducible using vertex reduction iff $G$ is nonsingular and each connected component of $G$ has at least one loop.

\section{Proof:}

We follow a similar line of reasoning as in the proof of [7, Lemma 7] (see also [6, Lemma 13.8]).

The condition of nonsingularity is required for $A$ to be successfully reducible. Also, in order to apply vertex reduction we need a looped vertex, hence the condition is necessary. 
We now show that the condition is sufficient. As this proof is graph theoretical in nature, we consider the graph $G$ with $A=A(G)$. We introduce some additional notation. The neighbourhood $N_{G}(u)$ of $u$ in $G$ is split into two sets, $N_{G}^{1}(u)$ and $N_{G}^{0}(u)$, of vertices with and without loop, respectively.

Assume that $A$ is nonsingular, and that each component of $G$ has a looped vertex. Consider the set $S$ of looped vertices in $G$ that maximizes $\left|N_{G}^{0}(w)\right|$ among all looped vertices $w \in V$, i.e., with a maximal number of unlooped neighbours. Choose a vertex $u$ in $S$ that minimizes $\left|N_{G}^{1}(w)\right|$ among all $w \in S$, i.e., has a minimal number of looped neighbours.

We claim that vertex reduction on $u$ does not introduce a component that consists completely of unlooped vertices in $G /\{u\}$. Assume that it does. As the component does not yet exist in $G$, it must be introduced by local complementation and it must include a looped neighbour $v \in N_{G}^{1}(u)$ of $u$. After local complementation on $u$, vertex $v$ cannot have looped neighbours, so in $G$ the unlooped neighbours of $u$ are also connected to $v$. Thus $N_{G}^{0}(u) \subseteq N_{G}^{0}(v)$. By the definition of $S$ this must be an equality $N_{G}^{0}(u)=N_{G}^{0}(v)$, and consequently also $v$ belongs to $S$.

Assume that $v$ has a looped neighbour in $G$. That neighbour must also be connected to $u$, as otherwise $v$ would have a looped neighbour in $G /\{u\}$. Thus $N_{G}^{1}(v) \subseteq N_{G}^{1}(u)$, and by minimality in the choice of $u$ this must be an equality $N_{G}^{1}(v)=N_{G}^{1}(u)$. Hence, $N_{G}(v)=N_{G}(u)$.

Consider $u$ and $v$. They are adjacent and both looped, and have the same neighbourhood. Thus they have equal columns in the adjacency matrix $A$ of $G$. Thus $A$ is singular - a contradiction. Hence the claim holds.

Since each component of $A /\{u\}$ has at least one 1 on the diagonal and $A /\{u\}$ is nonsingular, the argument can be applied iteratively.

The proof of Theorem 3.2 shows that a "greedy" method is available to find a sequence of vertex reductions to successfully reduce a graph. This greedy method chooses in each step a vertex with a minimal number of looped neighbours among the looped vertices with a maximal number of unlooped neighbours, and applies a reduction to this vertex. It is easy to see that, by symmetry, the proof of Theorem 3.2 also holds if one chooses in each step a vertex with a maximal number of unlooped neighbours among the looped vertices with a minimal number of looped neighbours. In this way, we obtain an alternative greedy method to successfully reduce a graph.

Example 3.1. Graph $G$ from Example 2.1 contains exactly two looped vertices. Of these, $q$ has most unlooped neighbours. Graph $G /\{q\}$ contains also exactly two looped vertices, of which $p$ has most unlooped neighbours. Continuing in this way we find the successful vertex reduction $/\{q\} /\{p\} /\{r\} /\{s\}$ of $G$ in four steps. Note that, in this case, other strategies do not work, i.e., eventually lead to a nonempty graph without looped vertices.

Inversion through local complementation. If $G / X_{1} \cdots / X_{k}$ is a successful reduction of graph $G$, then $X_{1}, \ldots, X_{k}$ is a partition of $V$. As applicability of pivot $* X$ on a graph $G$ (i.e., whether or not $G * X$ is defined) depends only on $G[X]$, we conclude that $G * X_{1} * \cdots * X_{k}$ is defined, and equal to the inverse $G * V=G^{-1}$. Hence, if $G$ satisfies the conditions of Theorem 3.2, then the inverse of graph $G$ is obtainable using only local complementation. The converse does not hold: in case $G$ consists of a single edge on a pair of unlooped vertices, corresponding with the matrix $\left(\begin{array}{ll}0 & 1 \\ 1 & 0\end{array}\right)$, no local complement operation is defined, while the inverse is trivially obtained (we have $G=G^{-1}$ ). 
Let $\varphi=* X_{1} \cdots * X_{k}$ be a sequence of local complementations defined on a graph $G$ where $X_{1} \oplus$ $\ldots \oplus X_{k}=V$. Then $G \varphi=G * V=G^{-1}$ and each connected component of $G$ has at least one loop. Hence, by Theorem 3.2, $G$ is successfully reducible using vertex reduction iff such $\varphi$ exists. Consequently, by the above, $\varphi$ may be chosen such that $X_{1}, \ldots, X_{k}$ are mutually disjoint (recall that, unlike the Schur complement, vertices may be used several times in a sequence of pivots). We have a similar characterization for edge complementation: a graph $G$ is successfully reducible using edge reduction iff $\varphi=* X_{1} \cdots * X_{k}$ be a sequence of edge complementations defined on a graph $G$ where $X_{1} \oplus \cdots \oplus X_{k}=V$.

\section{Gene Assembly in Ciliates and Schur Complementation}

Ciliates are a large group of single-cell organisms, the name of which comes from their characteristic hair-like organelles, typically used for movement and sensation. Curiously, ciliates have two very different kinds of nuclei, the micronucleus (MIC) and the macronucleus (MAC). During a complex process called gene assembly the "scrambled" gene segments occurring in the MIC are recombined to obtain the fully functional "sorted" genes in the MAC. This transformation of single genes from their MIC form to their MAC form is formally modelled in a series of papers by Ehrenfeucht et al. (see, e.g., $[10,5,7,8]$ ), culminating in a book [6]. See [2] for a more recent overview of the "computational" aspects of gene assembly.

For the transformation process, two (almost) equivalent models are proposed, string based and graph based, each having three rewriting operations [5, 8]. It turns out that an even more abstract viewpoint can be taken, that of set systems, see [4]. Regarding the graph based model, it has been noted in [3] that two of the three operations are in fact the two elementary pivot operations (the so-called "positive rule" gpr is local complementation and the so-called "double rule" gdr is edge complementation) followed by the deletion of the vertices involved, i.e., the two elementary Schur complementations, vertex and edge reduction. The third operation called the "negative rule" gnr simply removes isolated unlooped vertices.

In [7] gene "patterns" are investigated, characterizing the structure of the strings that can be successfully reduced using the various subsets of the three types of reduction rules. In [6, Chapter 13] the same question was posed, but now also for graphs, which could only be partially answered. The cases resolved there, cited below as Corollary 4.2, all involve gnr, leaving open the three cases that do not involve gnr.

The results of the previous section can be trivially reformulated in terms of these graph rewriting rules, and cover exactly the cases without gnr. Note that item (3) below has been obtained in [3].

Corollary 4.1. Let $G$ be a graph. Then $G$ can be successfully reduced using

(1) gdr iff $G$ is nonsingular and has no looped vertices,

(2) gpr iff $G$ is nonsingular and each connected component of $G$ has at least one looped vertex, and

(3) gpr and gdr iff $G$ is nonsingular.

Starting with a graph $G$, not necessarily nonsingular, a vertex reduction (gpr) or a edge reduction (gdr) is applicable as long as the graph has at least one loop or at least one edge. When these operations can no longer be performed, the resulting graph must consist of a number of isolated unlooped vertices. The number of these vertices is equal to the nullity of the original graph as Schur complement retains 
the nullity of the original matrix. The applicability of the gnr operation is exactly restricted to isolated unlooped vertices. As a consequence, as observed in [4], the number of gnr-operations in any successful reduction of graph $G$ is equal to the nullity of $G$.

These new characterizations can be contrasted to those involving gnr that were obtained before. We formulate them here, originally shown in [6, Section 13.3] for so-called overlap graphs (also called circle graphs) where loops are allowed. Although overlap graphs are a natural restriction in the context of gene assembly, this restriction is not needed in the proof of the results quoted here.

A connected component is called nontrivial if it contains at least one edge.

Corollary 4.2. Let $G$ be a graph. Then $G$ can be successfully reduced using

(4) gnr iff $G$ has no edge (i.e., consists of only isolated unlooped vertices),

(5) gnr and gdr iff $G$ has no looped vertices,

(6) gnr and gpr iff each nontrivial connected component of $G$ has at least one looped vertex, and

(7) gnr, gpr and gdr.

Reconsidering these previous results we see that adding the gnr operation essentially means omitting the nonsingularity requirement in the characterizations of Corollary 4.1. Hence, even to formulate the new results we are forced to move from graphs and their operations to the linear algebra of matrices and determinants over $\mathbb{F}_{2}$. More importantly, within the domain of matrices the interplay of gpr and gdr is much better understood than their formulation as graph operations: imagine Figure 1 when extended to cover two consecutive pivots. It is quite surprising that our arguments are nevertheless combinatorial in nature, and not algebraic.

Acknowledgements. We thank three kind referees for sharing their comments on our paper.

\section{References}

[1] Bouchet, A.: Representability of $\Delta$-matroids, Proc. 6th Hungarian Colloquium of Combinatorics, Colloquia Mathematica Societatis János Bolyai, 52, North-Holland, 1987.

[2] Brijder, R., Daley, M., Harju, T., Jonoska, N., Petre, I., Rozenberg, G.: Computational Nature of Gene Assembly in Ciliates, in: Handbook of Natural Computing (G. Rozenberg, T. Bäck, J. Kok, Eds.), Springer, 2011.

[3] Brijder, R., Harju, T., Hoogeboom, H.: Pivots, Determinants, and Perfect Matchings of Graphs, 2008, Submitted, [arXiv:0811.3500].

[4] Brijder, R., Hoogeboom, H.: Maximal Pivots on Graphs with an Application to Gene Assembly, Discrete Applied Mathematics, 158(18), 2010, 1977-1985, ISSN 0166-218X.

[5] Ehrenfeucht, A., Harju, T., Petre, I., Prescott, D., Rozenberg, G.: Formal systems for gene assembly in ciliates, Theoretical Computer Science, 292, 2003, 199-219.

[6] Ehrenfeucht, A., Harju, T., Petre, I., Prescott, D., Rozenberg, G.: Computation in Living Cells - Gene Assembly in Ciliates, Springer Verlag, 2004.

[7] Ehrenfeucht, A., Harju, T., Petre, I., Rozenberg, G.: Characterizing the micronuclear gene patterns in ciliates, Theory of Computing Systems, 35, 2002, 501-519. 
[8] Ehrenfeucht, A., Petre, I., Prescott, D., Rozenberg, G.: String and graph reduction systems for gene assembly in ciliates, Mathematical Structures in Computer Science, 12, 2002, 113-134.

[9] Geelen, J.: A generalization of Tutte's characterization of totally unimodular matrices, Journal of Combinatorial Theory, Series B, 70, 1997, 101-117.

[10] Prescott, D., Ehrenfeucht, A., Rozenberg, G.: Molecular operations for DNA processing in hypotrichous ciliates, European Journal of Protistology, 37, 2001, 241-260.

[11] Tsatsomeros, M.: Principal pivot transforms: properties and applications, Linear Algebra and its Applications, 307(1-3), 2000, 151-165.

[12] Tucker, A.: A combinatorial equivalence of matrices, Combinatorial Analysis, Proceedings of Symposia in Applied Mathematics, X, American Mathematical Society, 1960.

[13] Zhang, F.: The Schur Complement and Its Applications, Springer, 2005. 